Marco Aurélio B. Andrade

marcobrizzotti@gmail.com

\author{
Nicolas Pérez Alvarez \\ nperez@usp.br \\ Flávio Buiochi \\ Member, ABCM \\ fbuiochi@usp.br \\ University of São Paulo - USP \\ Escola Politecnica \\ 05508-030 São Paulo, SP, Brazil \\ Carlos Negreira \\ carlosn@fisica.edu.uy \\ Universidad de la República \\ Uruguay \\ Julio Cezar Adamowski \\ Member, ABCM \\ jcadamow@usp.br \\ University of São Paulo - USP \\ Escola Politecnica \\ 05508-030 São Paulo, SP, Brazil
}

\section{Analysis of 1-3 Piezocomposite and Homogeneous Piezoelectric Rings for Power Ultrasonic Transducers}

Some power ultrasonic transducers, such as Tonpilz transducers, require high-power transmitting capability as well as broadband performance. Optimized vibrational modes can achieve these requirements. This work compares the resonant characteristics and the surface vibration modes between a homogeneous piezoelectric ring and a 1-3 piezocomposite ring, both used in power ultrasonic transducers. This is the first step in the design of power transducers. Analytical models and finite element results are validated by electrical impedance measurements and the surface acoustic spectroscopy method. Excellent agreement between theoretical and experimental results was obtained. Results show that using piezocomposite ceramics minimize superposition of undesirable modes and increase the bandwidth, as shown in sonograms.

Keywords: transducer characterization, piezocomposite, underwater acoustic

\section{Introduction}

Conventional sandwich transducers consist of a stack of piezoelectric rings mounted between two masses (tail mass and head mass) prestressed by a central bolt. Placing a stack of piezoceramic rings between massive ends diminishes the operation frequency. The main characteristics of these transducers are low frequency (near 20 $\mathrm{kHz}$ ) and high power acoustic wave transmitted to the medium. As typical resonators, sandwich transducers have a straight bandwidth.

When there is need for high resolution, such as in sonar applications, broadband transducers are required (Tonpilz transducer). The bandwidth of the transducer can be improved by using a larger soft head (Yao and Bjorno, 1997). A soft rubber cone in front of the head mass increases the front diameter thus enhancing the acoustic impedance matching. Furthermore, the correct choice of the piezoelectric ring can also contribute to increase the bandwidth of the ultrasonic transducer. In order to choose the correct piezoelectric ring, it is important to understand the ring vibrational behavior.

In a piezoelectric ring there are three different vibration modes (Cheng and Chan, 2001): thickness, radial and wall thickness. To achieve a good performance, the piezoelectric ring used in the ultrasonic transducer should vibrate in the thickness mode. However, as the internal and external diameters are of the same order of thickness, there is a coupling between the thickness mode and the undesirable radial and wall thickness modes. This produces degradation of the transducer and has a negative influence on its performance (Yao and Bjorno, 1997; Or and Chan, 2001; Chong et al., 2005).

To reduce the mode coupling between these vibration modes, Or and Chan (2001) suggested the use of piezoelectric composite rings in the transducer construction. The piezoelectric composite material

Paper accepted May, 2009. Technical Editor: Domingos A. Rade consists of a combination of a piezoelectric material with a nonpiezoelectric polymer (Skinner et al., 1978; Akdogan et al., 2005). In comparison with piezoceramics, piezoelectric composites exhibit high electromechanical coupling factor, low acoustic impedance and low radial coupling (Smith and Auld, 1991). These characteristics allow the construction of broadband ultrasonic transducers with high sensitivity and the operation in the thickness mode without mode coupling (Roh, 2006). Another advantage of piezoelectric composites is the plate wave damping. Due to the plate wave damping, the normal velocity distribution along the transducer face can be considered uniform (Cathignol et al., 1999).

The aim of this paper is to analyze the performance of 1-3 piezoelectric composite rings and to compare its performance with a conventional piezoelectric ring. The paper presents an experimental and a theoretical analysis for both homogeneous and piezocomposite rings. The manufacturing technique of piezocomposite rings is also described.

\section{Nomenclature}

$A=$ area of the piezoelectric surface, $m^{2}$

$C_{i j} \quad=$ stiffness of the polymer, $\mathrm{N} / \mathrm{m}^{2}$

$c_{i j}^{E}=$ stiffness at constant electric field, $\mathrm{N} / \mathrm{m}^{2}$

$\bar{c}_{33}^{E}=$ homogenized stiffness at constant electric field, $\mathrm{N} / \mathrm{m}^{2}$

$e_{i j} \quad=$ piezoelectric constant, $\mathrm{C} / \mathrm{m}^{2}$

$\bar{e}_{33} \quad=$ homogenized piezoelectric constant, $\mathrm{C} / \mathrm{m}^{2}$

$f_{L 1}=$ frequency of the first lateral mode, $\mathrm{Hz}$

$f_{L 2}=$ frequency of the second lateral mode, $\mathrm{Hz}$

$f_{r} \quad=$ frequency of the radial mode, $\mathrm{Hz}$

$f_{t} \quad=$ frequency of the thickness mode, $\mathrm{Hz}$

$f_{w}=$ frequency of the wall thickness mode, $\mathrm{Hz}$ 


$$
\begin{array}{ll}
k & =\text { wavenumber } m^{-1} \\
l & =\text { thickness of the piezoelectric element, } m \\
Z^{e} & =\text { electrical impedance, } \Omega
\end{array}
$$

\section{Greek Symbols}

$$
\beta \quad \text { = damping, } \mathrm{s}
$$$$
\delta \quad=\text { volume fraction of piezoelectric ceramic, dimensionless }
$$$$
\varepsilon_{0} \quad=\text { permittivity of free space, } \mathrm{F} / \mathrm{m}
$$$$
\varepsilon_{i j}^{S} \quad=\text { permittivity at constant strain, } \mathrm{F} / \mathrm{m}
$$$$
\bar{\varepsilon}_{33}^{S}=\text { homogenized permittivity at constant strain, F/m }
$$$$
\rho^{c}=\text { density of the piezoelectric ceramic, } \mathrm{Kg} / \mathrm{m}^{3}
$$$$
\rho^{p} \quad=\text { density of the polymer, } \mathrm{Kg} / \mathrm{m}^{3}
$$$$
\bar{\rho} \quad=\text { homogenized density of the piezocomposite, } \mathrm{Kg} / \mathrm{m}^{3}
$$$$
\omega=\text { angular frequency, } \mathrm{rad} / \mathrm{s}
$$

\section{Fabrication of a 1-3 Connectivity Piezoelectric Composite Ring}

In this work, the dice-and-fill technique (Smith, 1989; Savakus et al., 1981) is used to fabricate the 1-3 piezoelectric composite ring, which is illustrated in Fig. 1. First, a PZT-8 piezoelectric ring of 7.6 $\mathrm{mm}$ internal diameter, $27.4 \mathrm{~mm}$ external diameter and $5.1 \mathrm{~mm}$ thickness is cut using a dicing machine (Buehler Isomet 4000) with a $150 \mu \mathrm{m}$-thick blade. The grooves on the ceramic are filled with epoxy (GY 279 with hardener HY951 in a mixing ratio of 10:1, supplied by Huntsman). To avoid air bubbles, this epoxy is degassed in a vacuum chamber for approximately 5 minutes. Then, ceramic with polymer is put in an oven for 2 hours at a temperature of $50{ }^{\circ} \mathrm{C}$. After that, the composite is cured at room temperature for 24 hours. A sandpaper is used to remove the polymer excess of the piezoelectric composite material. After removing the polymer excess, the electrodes of the composite are made using a conductive silver ink.

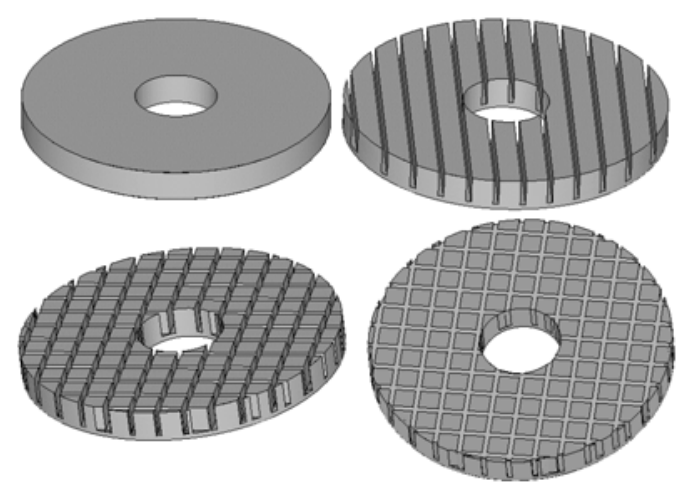

Figure 1. Fabrication of a 1-3 piezoelectric composite ring using the diceand-fill technique.

The unit cell of this composite is shown in Fig. 2. This composite has a ceramic volume fraction of $88.4 \%$ because, as the transducer is for power application, it requires a high volume of piezoelectric ceramic. The piezoelectric composite can be modeled by considering that the material is homogeneous. According to Smith and Auld (Smith and Auld, 1991), the thickness mode behavior can be easily modeled by considering that the aspect ratio (ratio between the thickness and the lateral dimension of the unit cell) is much greater than 1. Hayward and Bennett (Hayward and
Bennett, 1996) showed that an aspect ratio above 2 is sufficient to model the thickness mode in a composite with $88.4 \%$ ceramic volume fraction. In this work, the aspect ratio corresponds to 2.04 and therefore this condition is satisfied.

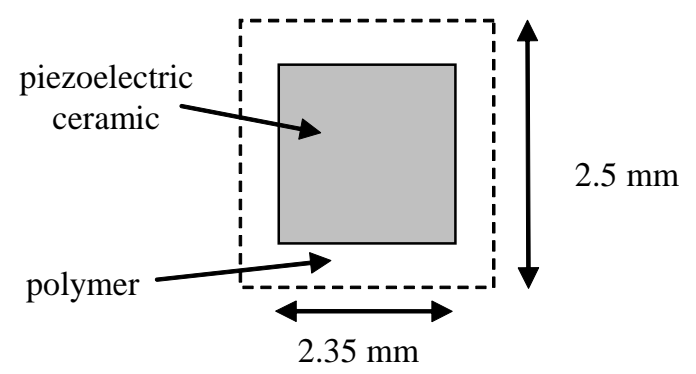

Figure 2. Unit cell of the piezoelectric composite.

\section{Analytical and Numerical Modeling of a Homogeneous Piezoelectric Ring}

First, a unidimensional model is presented to describe the thickness behavior of a homogeneous piezoelectric ring. The ring used in this work is polarized along its thickness. The polarization axis is aligned with direction 3 . The unidimensional model assumes that lateral dimensions of the ring are much larger than its thickness. The theoretical electrical impedance $Z^{e}$ of the piezoelectric ring is given by (Kino, 1987):

$$
Z^{e}=\left(\frac{l}{j \omega A \varepsilon_{33}^{S}}\right)\left[1-\left(1-\frac{c_{33}^{E}}{c_{33}^{E}+\frac{\left(e_{33}\right)^{2}}{\varepsilon_{33}^{S}}}\right) \frac{\tan \left(\frac{k l}{2}\right)}{\left(\frac{k l}{2}\right)}\right]
$$

where $A$ is the area of the piezoelectric surface, $l$ is the thickness, $\omega$ is the angular frequency. The variables $c_{33}^{E}, e_{33}$ and $\varepsilon_{33}^{S}$ are, respectively, the elastic stiffness constant at constant electric field, the piezoelectric constant, and the permittivity at constant strain of the piezoelectric ring. The wavenumber $k$ is given by:

$$
k=\omega \sqrt{\frac{\rho^{c}}{c_{33}^{E}+\frac{\left(e_{33}\right)^{2}}{\varepsilon_{33}^{s}}}}
$$

where $\rho^{c}$ is the density of the ring.

The unidimensional model used describes only the thickness mode of the piezoelectric ring. In order to simulate all the vibrational modes of the piezoelectric ring, finite element method is used (Lerch, 1990; Naillon and Besnier, 1970). The piezoelectric ring used in this work is made of lead zirconate titanate (PZT-8). Due to the circular geometry of the ring, axisymmetric elements are used. This allows the reduction of a three-dimensional analysis to a two-dimensional one. The ring modeling is obtained by using the ANSYS commercial package. The material properties of the PZT-8 (Vernitron, 1976) are presented in Table 1 , where $c_{i j}^{E}$ is the elastic stiffness constant at constant electric field, $e_{i j}$ is the piezoelectric constant, $\varepsilon_{i j}^{s} / \varepsilon_{0}$ is the dielectric constant at constant strain, where $\varepsilon_{0}$ $=8.85 \times 10^{-12} \mathrm{~F} / \mathrm{m}$ is the permittivity of free space and $\rho^{c}$ is the 
density. The damping coefficient $\beta$ is not provided by the manufacturer and in this work it was chosen to match the experimental results. In the ANSYS package, harmonic analysis is used to determine the electrical impedance as a function of frequency. The comparison between the electrical impedance curves obtained by the finite element method and by the unidimensional model is shown in Fig. 3. In order to show the influence of the piezoelectric ring diameter on the coupling between the radial and wall thickness modes with the thickness mode, two different simulations were performed. In Fig. 3(a), the ring has an external diameter of $27.4 \mathrm{~mm}$, internal diameter of $7.6 \mathrm{~mm}$ and a thickness of $5.1 \mathrm{~mm}$. In Fig 3(b), the ring has an external diameter of $160 \mathrm{~mm}$, an internal diameter of $20 \mathrm{~mm}$ and a thickness of $5.1 \mathrm{~mm}$. As it is seen in Fig. 3, the unidimensional model can predict only the thickness mode of the piezoelectric ring. In Fig. 3(a), as the thickness of the piezoelectric ring is not much larger than its radius, there is mode coupling between the thickness mode and the radial modes. In Fig. 3(b), as the radius of the ring is much larger than its thickness, the unidimensional model can be used to predict the thickness behavior of the piezoelectric ring.

Table 1. Material properties of PZT-8.

\begin{tabular}{cc}
\hline \hline piezoelectric ceramic & PZT-8 \\
\hline$c_{11}^{E}\left(10^{10} \mathrm{~N} / \mathrm{m}^{2}\right)$ & 13.7 \\
$c_{12}^{E}\left(10^{10} \mathrm{~N} / \mathrm{m}^{2}\right)$ & 6.97 \\
$c_{13}^{E}\left(10^{10} \mathrm{~N} / \mathrm{m}^{2}\right)$ & 7.16 \\
$c_{33}^{E}\left(10^{10} \mathrm{~N} / \mathrm{m}^{2}\right)$ & 12.4 \\
$c_{44}^{E}\left(10^{10} \mathrm{~N} / \mathrm{m}^{2}\right)$ & 3.14 \\
$e_{31}\left(\mathrm{C} / \mathrm{m}^{2}\right)$ & -4.0 \\
$e_{33}\left(\mathrm{C} / \mathrm{m}^{2}\right)$ & 13.8 \\
$e_{15}\left(\mathrm{C} / \mathrm{m}^{2}\right)$ & 10.4 \\
$\varepsilon_{11}^{S} / \varepsilon_{0}$ & 898 \\
$\varepsilon_{33}^{S} / \varepsilon_{0}$ & 582 \\
$\rho^{c}\left(\mathrm{Kg} / \mathrm{m}^{3}\right)$ & 7600 \\
$\beta\left(10^{-9} \mathrm{~s}\right)$ & 2 \\
\hline \hline
\end{tabular}

The resonance frequencies of the main vibrational modes presented in Fig. 3(a) are denoted by $f_{r}$ for the first radial mode $(68.3 \mathrm{kHz}), f_{w}$ for the first wall thickness mode $(194.0 \mathrm{kHz})$, and $f_{t}$ for the thickness mode $(428.6 \mathrm{kHz})$. The vibration modes of the piezoelectric ring can be observed in Fig. 4. The first radial mode is shown in Fig. 4(b). In this vibration mode, the inner and outer walls of the ring vibrate in phase. In the wall thickness mode shown in Fig. 4(c), the inner and outer walls vibrate in opposite phase. In the thickness mode of the ring, the displacements of the upper and lower surfaces are in opposite phase. In Fig. 4(d), there is mode coupling between the first thickness mode and the harmonics of the radial and wall thickness modes. This mode coupling is responsible for the non-uniform displacement of the surfaces of the ring.
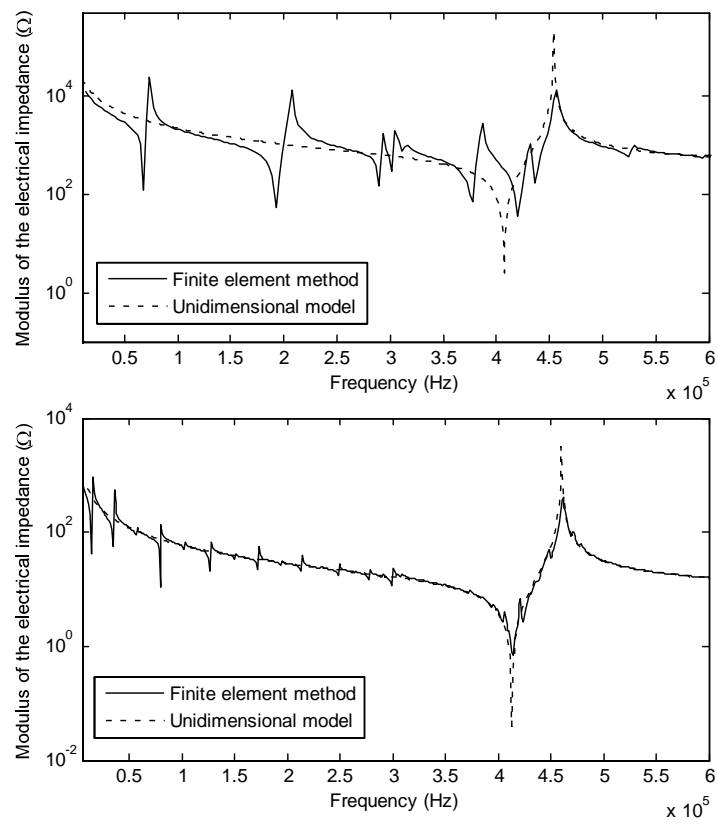

Figure 3. Comparison between the electrical impedance of the homogeneous piezoelectric ring determined by the finite element method and by the unidimensional model: (a) external diameter of $27.4 \mathrm{~mm}$; (b) external diameter of $160 \mathrm{~mm}$.

\section{Experimental Determination of the Vibrational Modes of the Piezoelectric Ring}

In order to validate the vibrational modes determined by the finite element method, it is necessary to measure the surface displacement of piezoelectric rings. Usually, the surface displacements are determined through laser probe measurements (Gururaja et al., 1985). In this work, the surface acoustic spectroscopy is used (Perez, 2002). Contrary to the laser probe measurement, this technique does not measure the absolute value of the displacements, but the relative ones. The advantage of the surface acoustic spectroscopy over the laser probe measurements is its possibility to measure the relative displacement in each point as a function of the frequency, also showing the position over the surface. The surface acoustic spectroscopy setup is shown in Fig. 5. This technique provides a graphic called sonogram.

Before showing the sonogram of the piezoelectric ring, the simulated electrical impedance of the ring is compared with the one obtained experimentally by the HP4194A impedance analyzer. The comparison between the experimental and simulated electrical impedances is shown in Fig. 6. There is excellent agreement between the experimental electrical impedance and that obtained by the finite element method, as shown in Fig. 6. As previously predicted by the finite element method, the experimental electrical impedance curve shows that there is a mode coupling between the first thickness mode $\left(f_{t}=428.6 \mathrm{kHz}\right)$ and the harmonics of the radial and wall thickness modes. 
a)<smiles>[Z]C[Z]</smiles>

b)<smiles>[Z]C[Z]</smiles>

c)

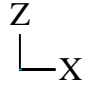

d) $\mathrm{Z}$

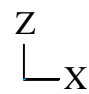

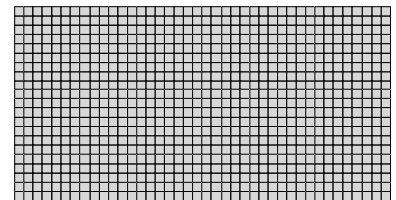
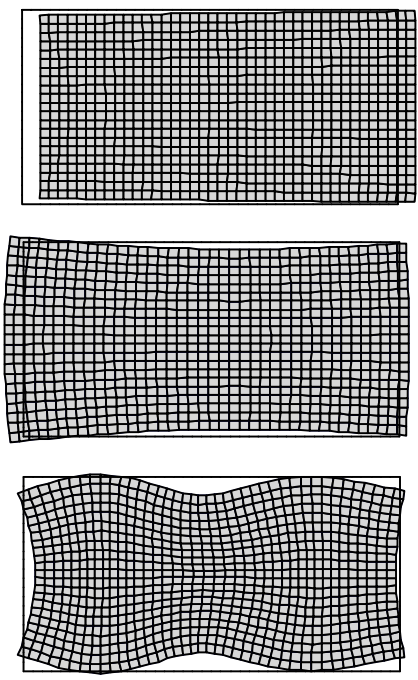

Figure 4. Vibrational modes of the homogeneous piezoelectric ring: (a) nondeformed; (b) radial mode; (c) wall thickness mode; (d) thickness mode.

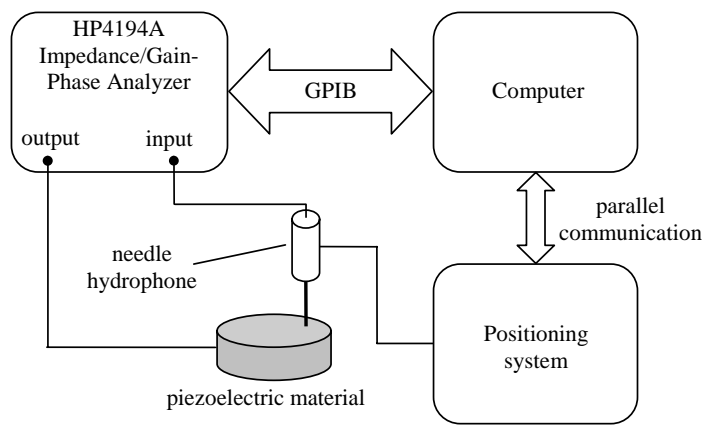

Figure 5. Surface acoustic spectroscopy setup.

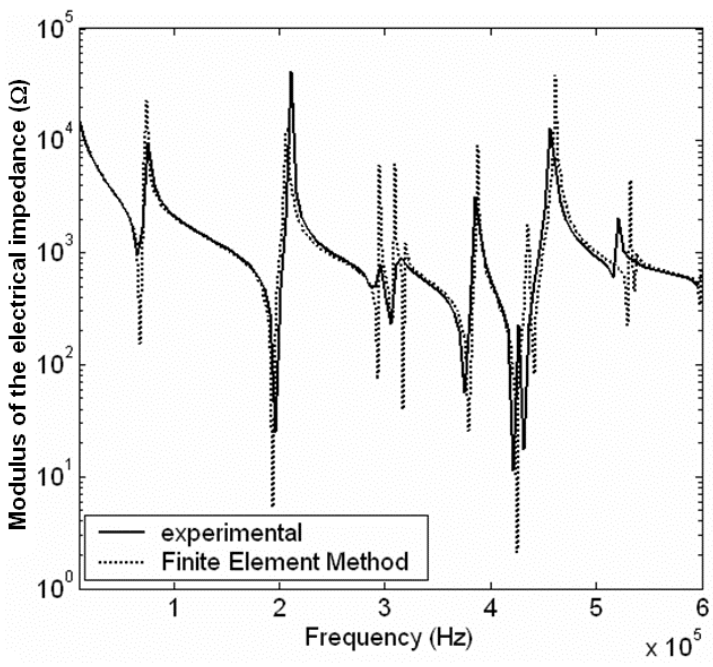

Figure 6. Comparison between the experimental and simulated electrical impedance.
Figure 7 shows the comparison between the experimental and simulated sonogram. In order to enhance weak displacement amplitudes, the sonogram was plotted in a logarithmic scale. In the sonogram, the frequencies of the white vertical lines agree with the resonance frequencies from the electrical impedance curve.

(a)

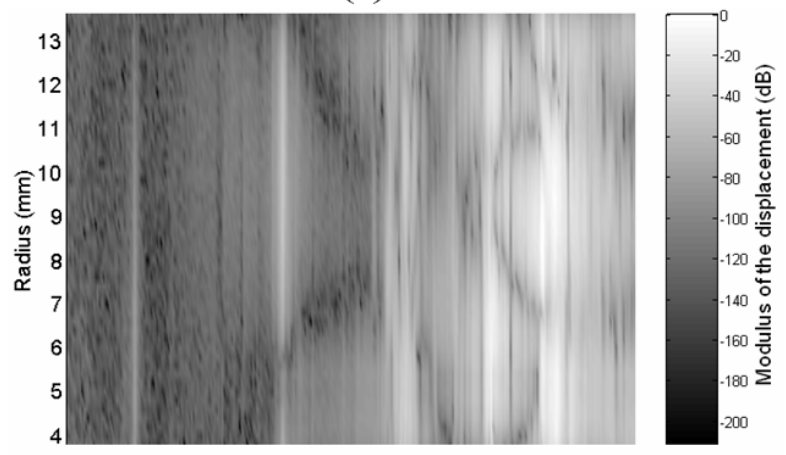

(b)

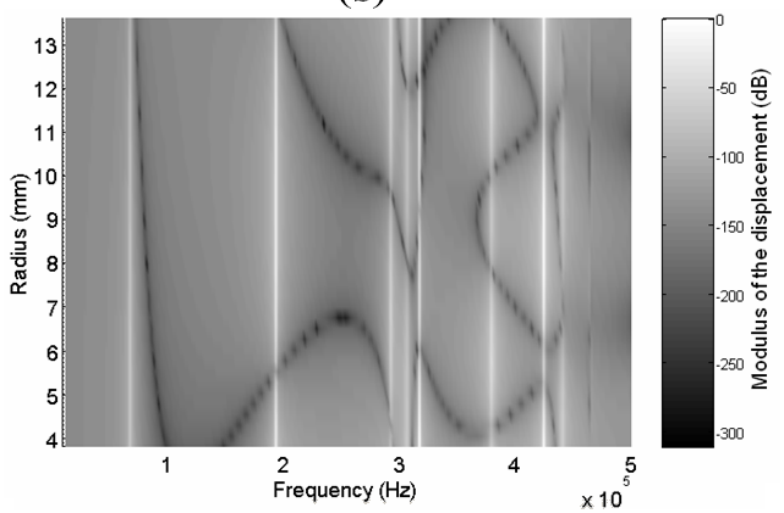

Figure 7. Comparison between the experimental and simulated sonogram: (a) experimental sonogram; (b) simulated sonogram.

Figure 8 shows the comparison between the simulated and experimental surface displacement profiles for the radial, wall thickness and thickness modes of the piezoelectric ring. The radial mode shown in Fig. 8(a) corresponds to the first white vertical line $\left(f_{r}=68.3 \mathrm{kHz}\right)$ in Fig. 7. The wall thickness and thickness modes shown in Figs. 8(b) and 8(c) correspond to the white vertical lines of frequencies $194.0 \mathrm{kHz}$ and $428.6 \mathrm{kHz}$ respectively, shown in Fig. 7. As the surface acoustic spectroscopy setup measures relative values of the displacements, the experimental displacements are normalized to fit to the simulated ones. Figure 8 shows good agreement between the experimental and simulated results, especially for the radial and wall thickness modes. As it can be seen in Figs. 6 and 7, there is no mode superposition at these two frequency ranges. In these cases, it is easier to model the displacements of these two modes. In displacement profile of Fig. 8(c), there is mode coupling between the thickness mode and the harmonics of the radial and wall thickness modes. When there is mode coupling, small changes in the geometry and material properties can lead to a completely different displacement profile. 


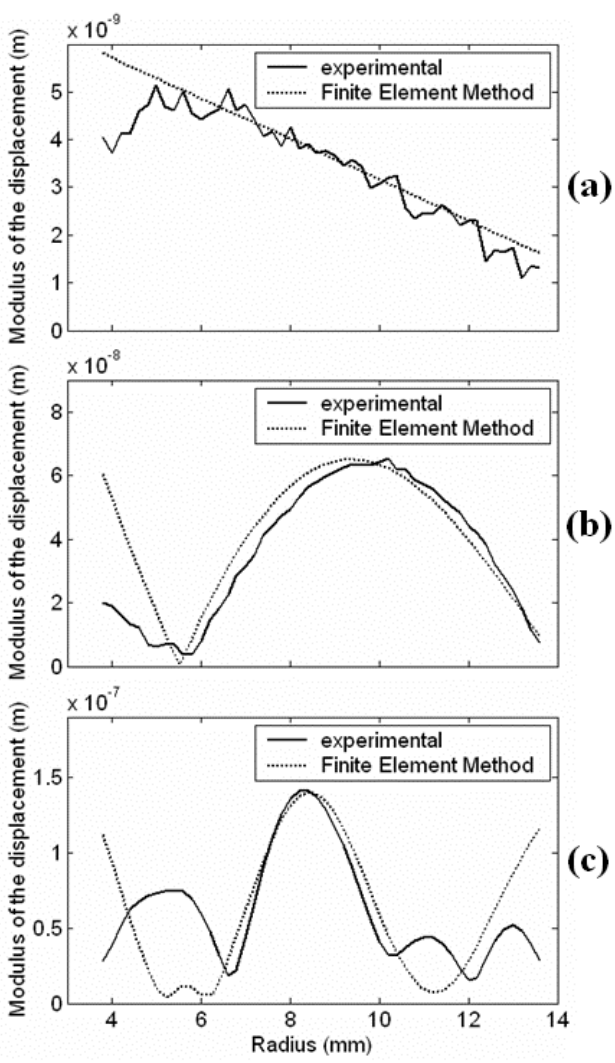

Figure 8. Surface displacement profiles of the piezoelectric ring: (a) radial mode; (b) wall thickness mode; (c) thickness mode.

\section{Analytical and Numerical Modeling of a Piezoelectric Composite Ring}

The modeling of the piezoelectric composite can be made by the unidimensional model and the finite element method. The unidimensional model assumes that the piezoelectric composite can be treated as a homogeneous medium. Thus, it is necessary to calculate the effective properties of the composite in order to use equation (1) to determine the electrical impedance. In Eq. (1), the properties $c_{33}^{E}, e_{33}$ and $\varepsilon_{33}^{S}$ are replaced by the properties $\bar{c}_{33}^{E}, \bar{e}_{33}$ and $\bar{\varepsilon}_{33}^{s}$, which represent the homogenized properties of the composite. These properties are given by (Smith and Auld, 1991):

$$
\begin{aligned}
& \bar{c}_{33}^{E}=\delta\left[c_{33}^{E}-\frac{2(1-\delta)\left(c_{13}^{E}-c_{12}\right)^{2}}{\delta\left(c_{11}+c_{12}\right)+(1-\delta)\left(c_{11}^{E}+c_{12}^{E}\right)}\right]+(1-\delta) c_{11} \\
& \bar{e}_{33}=\delta\left[e_{33}-\frac{2(1-\delta) e_{31}\left(c_{13}^{E}-c_{12}\right)}{\delta\left(c_{11}+c_{12}\right)+(1-\delta)\left(c_{11}^{E}+c_{12}^{E}\right)}\right] \\
& \bar{\varepsilon}_{33}^{S}=\delta\left[\varepsilon_{33}^{S}+\frac{2(1-\delta)\left(e_{31}\right)^{2}}{\delta\left(c_{11}+c_{12}\right)+(1-\delta)\left(c_{11}^{E}+c_{12}^{E}\right)}\right]+(1-\delta) \varepsilon_{11}
\end{aligned}
$$

where $\delta$ is the volume fraction of piezoelectric ceramic. In Eqs. (3), (4) and (5), the elastic and dielectric constants of the ceramic phase are distinguished from those of the polymer phase by the superscripts $E$ and $S$. The model used to determine the homogenized properties is valid for any ceramic volume fraction and it is assumed that the aspect ratio of the unit cell is much higher than 1. In Eq. (2) the density $\rho^{c}$ should be replaced by the effective density $\bar{\rho}$ that is given by the mixture rule:

$$
\bar{\rho}=\delta \rho^{c}+(1-\delta) \rho^{p}
$$

where $\rho^{c}$ is the density of the piezoelectric material and $\rho^{p}$ is the density of the polymer.

As the piezoelectric composite has a lateral periodic structure, Bragg diffraction can occur. This phenomenon leads to a lateral vibration mode. The lateral modes (Certon et al., 1997) of the piezoelectric composite cannot be predicted by the unidimensional model. In this work, these modes are predicted by the finite element method. As the composite has a periodic structure, the simulation is performed in a single unit cell of the piezoelectric composite. Due to the symmetry of the unit cell, only one-eighth of it is modeled, as shown in Fig. 10(a). As boundary conditions, the displacements in the normal directions to the lateral and lower surfaces of the unit cell are set to zero. The thickness of the electrodes is thin and therefore, it can be neglected in the finite element analysis. An electrical potential difference of $0.5 \mathrm{~V}$ between the upper and lower surfaces is applied. The properties of the piezoelectric material are shown in Table 1, and the properties of the polymer are presented in Table 2. The electrical impedance of the composite is determined by a harmonic analysis in ANSYS package and it is compared with the one obtained by the unidimensional model. The comparison between the electrical impedances is shown in Fig. 9. As it can be seen in this figure, the unidimensional model cannot predict the lateral modes of the piezoelectric composite. In Fig. 9, the first and second lateral modes are denoted by $f_{L 1}$ and $f_{L 2}$, respectively. The peak associated with the first lateral mode cannot be clearly seen in the electrical impedance curve. The resonance frequency is $804 \mathrm{kHz}$ for the first lateral mode and $912 \mathrm{kHz}$ for the second lateral mode. The unidimensional model does not account for losses and, therefore, the peak associated with the harmonic of the thickness mode (around $1.25 \mathrm{MHz}$ ) is much more pronounced in the unidimensional model than in the finite element method.

Table 2. Mechanical properties of the epoxy GY 279 with hardener HY951.

\begin{tabular}{cc}
\hline \hline polymer & GY279/HY951 \\
\hline$c_{11}\left(10^{10} \mathrm{~N} / \mathrm{m}^{2}\right)$ & 0.704 \\
$c_{12}\left(10^{10} \mathrm{~N} / \mathrm{m}^{2}\right)$ & 0.422 \\
$\rho^{p}\left(\mathrm{Kg} / \mathrm{m}^{3}\right)$ & 1126 \\
$\beta\left(10^{-8} \mathrm{~s}\right)$ & 2 \\
\hline \hline
\end{tabular}

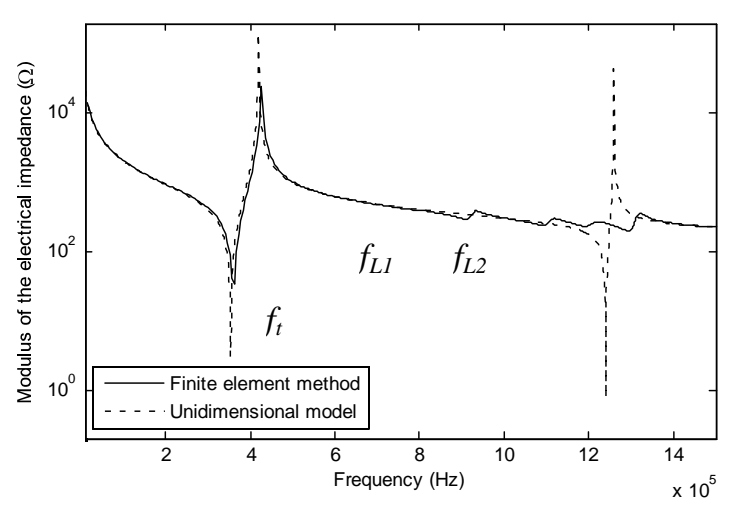

Figure 9. Comparison between the electrical impedance of the piezoelectric composite ring determined by finite element method and by the unidimensional model. 
The vibrational modes of the piezoelectric composite are shown in Fig. 10. The thickness mode $\left(f_{t}=362 \mathrm{kHz}\right)$ of the composite is shown in Fig. 10(b). As it can be seen in this figure, the surface displacements are almost uniform for this vibrational mode. The lateral modes of the composite are shown in Figs. 10(c) and 10(d). To avoid mode coupling between the first lateral mode and the first thickness mode, the aspect ratio (ratio between the thickness and the lateral dimension of the unit cell) of the composite should be sufficiently high. As this condition is satisfied in this composite, there is no mode coupling in the thickness mode. (a)

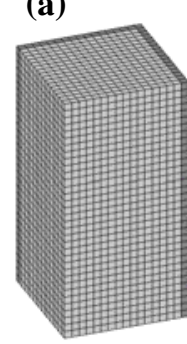

(b)

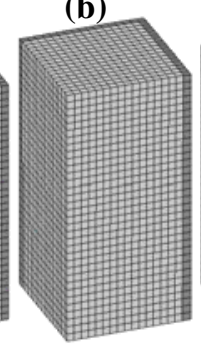

(c)

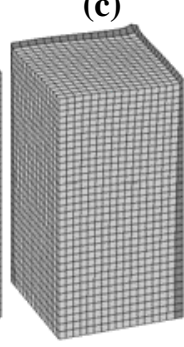

(d)

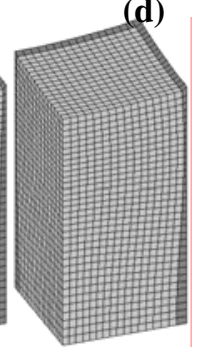

Figure 10. Vibrational modes of the piezoelectric composite: (a) nondeformed; (b) thickness mode; (c) first lateral mode; (d) second lateral mode.

\section{Experimental Determination of the Vibrational Modes of the Piezoelectric Composite Ring}

The electrical impedance of the piezoelectric composite ring was measured through the HP4194A impedance analyzer. The comparison between the experimental impedance curve and those obtained by finite element method is shown in Fig. 11. When comparing the experimental results of electrical impedance of the piezoelectric composite ring with the one of the homogeneous piezoelectric ring (Fig. 6), it can be observed that the amplitudes of the resonance peaks associated with the radial and wall thickness modes of the piezoelectric composite ring are lower than those of the homogeneous piezoelectric ring. In the homogeneous piezoelectric ring, the harmonics of the radial and thickness modes interfere with the thickness mode of the ring, causing a nonuniform velocity distribution of the ring surfaces. In the piezoelectric composite, there is a reduction of the radial and wall thickness modes, generating a smoother impedance curve. Due to the boundary conditions used in finite element method, the radial and wall thickness modes of the composite cannot be predicted. A possible alternative to model the composite radial and wall thickness modes is to use the homogenization theory (Silva et al., 1999) to determine the composite homogenized properties. These properties could be used in the finite element method to simulate the piezoelectric composite, however, this procedure would not predict the composite lateral modes, since it considers that the piezoelectric composite is homogeneous. Comparing the electrical impedance curves of Figs. 6 and 11, the resonance frequency for the thickness mode of the composite is lower than the one of the homogeneous piezoelectric ring. This reduction of the resonance frequency in the composite can be explained by the determination of the effective properties of the composite (Smith and Auld, 1991). According to the model used by Smith and Auld to calculate the effective properties of the composite, the longitudinal velocity of the composite is lower than that of the homogeneous piezoelectric material. This reduction of the longitudinal velocity is responsible for the reduction of the resonance frequency of the piezoelectric composite.

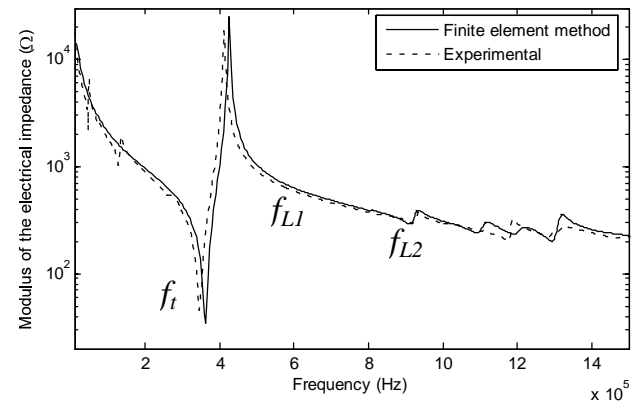

Figure 11. Comparison between the experimental and simulated electrical impedance of the piezoelectric composite ring.

Figure 13 shows the sonogram of the piezoelectric composite ring. The sonogram shown in Fig. 13(a) is obtained by the finite element method and the sonogram in Fig 13(b) is experimentally obtained through the surface acoustic spectroscopy technique. Contrary to Fig. 7, the relative displacements for the composite are not measured along the radial direction, but along six unit cells, as shown in Fig. 12. In Fig. 13(b), the peak associated with the thickness mode is more pronounced when compared with the radial and wall thickness modes. This phenomenon occurs due to the reduction of the radial and wall thickness modes in the composite, as observed in the electrical impedance curve. It can be observed in Fig. 13 that there is no mode coupling between the first thickness mode and the harmonics of the radial and wall thickness mode. However, in the frequency range around $1.25 \mathrm{MHz}$ there is mode coupling between the third harmonic of the thickness mode and the lateral vibration modes of the piezoelectric composite. This mode coupling does not affect the performance of the piezoelectric composite, since this composite was designed to operate in the first thickness mode.

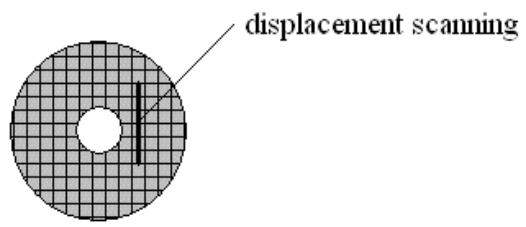

Figure 12. Diagram showing the displacement scanning position.

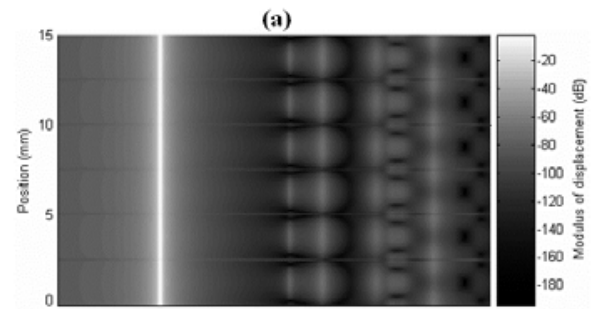

(b)

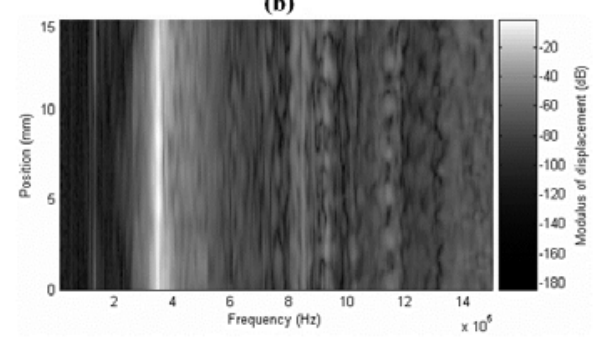

Figure 13. Comparison between the experimental and simulated sonogram for the piezoelectric composite ring: (a) simulated sonogram; (b) experimental sonogram. 


\section{Conclusion}

This paper presented the theoretical and experimental studies of the vibrational modes of homogeneous and 1-3 piezoelectric composite rings. The theoretical studies were performed using an analytical model and a finite element analysis, and the experimental studies were performed by measuring the electrical impedance of the rings and by using the surface acoustic spectroscopy method.

The analysis of the vibrational modes of a homogeneous piezoelectric ring showed that the radial and wall thickness modes can superpose with the thickness mode of the ring, causing a nonuniform vibration pattern of the ring surface. In comparison with a homogeneous piezoelectric ring, a piezoelectric composite ring has a lower mode coupling between the thickness mode and the radial and wall thickness modes. The reduction of the mode coupling is responsible for a more uniform vibration pattern of the ring surface.

Another difference between a homogeneous and a piezoelectric composite ring is its bandwidth. The bandwidth of a piezoelectric ring can be estimated by the width of the vertical lines in the sonogram. When the bandwidth of the ring is increased, the width of the vertical lines is also increased. Some applications, such as coded signal transmission and sonar, require broadband transducers.

As a future work, piezoelectric composite rings should be used in the construction of Tonpilz transducers. The performance of Tonpilz transducers constructed with composite rings should be compared with that a conventional Tonpilz transducer. It is expected that the performance of the transducer with the piezocomposite ring should be better than that with a homogeneous piezoelectric ring.

\section{Acknowledgements}

We would like to thank the following Brazilian sponsor agencies: CAPES, CNPq, FAPESP and FINEP for financial support.

\section{References}

Akdogan, E.K., Allahverdi, M., Safári, A., 2005, "Piezoelectric composites for sensor and actuator applications”, IEEE Transactions on Ultrasonics, Ferroelectrics, and Frequency Control, Vol. 52, No. 5, pp. 746-775.

Cathignol, D., Sapozhnikov, O.A., Theillère, Y., 1999, "Comparison of acoustic fields radiated from piezoceramic and piezocomposite focused radiators", Journal of the Acoustical Society of America, Vol. 105, No. 5, pp. 2612-2617.

Certon, D., Patat, F., Levassort, F., Feuillard, G., Karlsson, B., 1997, "Lateral resonances in 1-3 piezoelectric periodic composite: modeling and experimental results", Journal of the Acoustical Society of America, Vol. 101, No. 4, pp. 2043-2051.
Cheng, K.C., Chan, H.L.W., 2001, “Characterization of piezoelectric ring used for wire bonding transducer application”, Proceeding of the 2001 IEEE Electron Devices Meeting, pp. 64-67.

Chong, C.P., Chan, H.L.W., Chan, M.H., Liu, P.C.K., 2005, "Resonances in 1-3 piezocomposite rings", Applied Physics A: Materials Science \& Processing, Vol. 80, pp. 1793-1799.

Gururaja, T.R., Schulze, W.A., Cross, L.E., Newnham, R.E., Auld, B.A., Wang, Y.J., 1985, "Piezoelectric composite materials for ultrasonic transducer applications. Part I: resonant modes of vibration of PZT rod-polymer composites”, IEEE Transactions on Sonics and Ultrasonics, Vol. 32, No. 4, pp. 481-498.

Hayward, G., Bennett, J., 1996, “Assessing the influence of pillar aspect ratio on the behaviour of 1-3 connectivity composite transducers", IEEE Transactions on Ultrasonics, Ferroelectrics, and Frequency Control, Vol. 43, No. 1, pp. 98-108.

Kino, G.S., 1987, “Acoustic Waves: Devices, Imaging, and Analog Signal Processing”, Prentice Hall, New Jersey, EUA, 601p.

Lerch, R., 1990, "Simulation of piezoelectric devices by two- and threedimensional finite elements", IEEE Transactions on Ultrasonics, Ferroelectrics, and Frequency Control, Vol. 37, No. 2, pp. 233-247.

Naillon, M., Besnier, F., 1970, “Analysis of piezoelectric structures by a finite element method”, Acta Electronica, Vol. 25, pp. 341-362.

Or, S.W., Chan, H.L.W., 2001, "Mode coupling in lead Zirconate Titanate/epoxy 1-3 piezocomposite rings”, Journal of Applied Physics, Vol. 90, No. 8, pp. 4122-4129.

Perez, N., 2002, "Dinámica de estructuras piezocomposites complejas: aplicación a transductores de ultrasonido”, Tesis de Maestria, Universidad de la Republica, Montevideo, Uruguay, 135 p.

Roh, Y., Lu, X., 2006, "Design of an underwater Tonpilz transducer with 2-2 mode piezocomposite materials". The Journal of the Acoustical Society of America, Vol. 119, No 6, pp. 3734-3740.

Savakus, H.P., Klicker, K.A., Newnham, R.E., 1981, "PZT-epoxy piezoelectric transducers: a simplified fabrication procedure”, Materials Research Bulletin, Vol. 16, No. 6, pp. 677-680.

Silva, E.C.N., Fonseca, J.S.O., Espinosa, F.M., Crumm, A.T., Brady, G.A., Halloran, J.W., Kikuchi, N.. 1999, "Design of piezocomposite materials and piezoelectric transducers using topology optimization - Part I", Archives of Computational Methods in Engineering, Vol. 6, No. 2, pp. 117-182.

Skinner, D.P., Newnham, R.E., Cross, L.E., 1978, "Flexible composite transducers”, Materials Research Bulletin, Vol. 13, No. 6, pp. 599-607.

Smith, W.A., 1989, "The role of piezocomposites in ultrasonic transducers", Proceeding of the 1989 IEEE Ultrasonic Symposium, pp. 755-766.

Smith, W.A., Auld, B.A., 1991, "Modeling 1-3 composite piezoelectrics: thickness-mode oscillations", IEEE Transactions on Ultrasonics, Ferroelectrics, and Frequency Control, Vol. 38, No. 1, pp. 40-47.

Vernitron Limited, 1976, "Measuring Properties of Piezoelectric Ceramics”, Bulletin 66011/B.

Yao, A., Bjorno, L., 1997, "Broadband Tonpilz underwater acoustic transducers based on multimode optimization", IEEE Transactions on Ultrasonics, Ferroelectrics, and Frequency Control, Vol. 44, No. 5, pp. 1060-1066. 